\title{
Entropy Errors in the Numerical Approximation of the Euler Flow along a Kinked Wall
}

\author{
Eric van der Maarel and Barry Koren \\ Centre for Mathematics and Computer Science \\ P.O. Box 4079, 1009 AB Amsterdam, The Netherlands
}

\begin{abstract}
Numerical entropy generation is studied in the case of steady, subsonic Euler flow along a kinked wall. For a standard upwind finite volume discretization, the numerical entropy error appears to be zeroth-order in mesh size. Two possible causes of the zeroth-order entropy error are studied. First an investigation is made of the local discretization error on a kinked grid. In the neighbourhood of the kink, this error appears to be zeroth-order as well. For supersonic flow it can be easily removed. However, it seems that the zeroth-order entropy error is yet not caused by the zeroth-order discretization error. Next a study is made of the existence of a singularity in the exact solution. Probably, the Euler flow solution is singular at the kink in the wall. The form of the likely singularity is unknown. Therefore, the construction of a computational method which uses a priori knowledge of the singularity is not possible. Finally it is shown by numerical experiment that the subsonic Euler flow along a kinked wall still can be computed with vanishing entropy errors, by using a continuously curved wall which converges to the kinked wall in the limit of zero mesh width.
\end{abstract}

Note: This work was supported by the European Space Agency (ESA), through Avions Marcel Dassault - Bréguet Aviation (AMD-BA).

\section{INTRODUCTION}

\subsection{Governing equations}

The flow of an inviscid, non-heat conducting, perfect gas is described by the Euler equations and the perfect gas law. The steady, two-dimensional Euler equations can be written as

$$
\frac{\partial f(q)}{\partial x}+\frac{\partial g(q)}{\partial y}=0
$$

with

$$
q=\left(\begin{array}{l}
\rho \\
\rho u \\
\rho v \\
\rho E
\end{array}\right), \quad f(q)=\left(\begin{array}{l}
\rho u \\
\rho u^{2}+p \\
\rho u v \\
\rho u H
\end{array}\right), \quad g(q)=\left[\begin{array}{l}
\rho v \\
\rho u v \\
\rho v^{2}+p \\
\rho v H
\end{array}\right) .
$$

In (1.2) the usual notations have been used: $u$ and $v$ for the velocity components in 
$x$ - and $y$-direction, and $\rho$ and $p$ for the density and pressure. $E$ denotes the total energy defined by $E=e+1 / 2\left(u^{2}+v^{2}\right)$, with $e$ the internal energy, which for a perfect gas can be written as

$$
e=\frac{1}{\gamma-1} \frac{p}{\rho}
$$

$\gamma$ denoting the ratio of specific heats. The total enthalpy, denoted by $H$, is defined by $H=E+p / \rho$. It can be shown now that for the entropy function $s=p / \rho^{\gamma}$, the steady Euler equations imply

$$
u \frac{\partial s}{\partial x}+v \frac{\partial s}{\partial y}=0
$$

which means that - except for possible discontinuities - the entropy is constant along a streamline.

\subsection{Discretization}

In this section we give a brief outline of the basic discretization method used in this paper: the upwind finite volume method which was first presented in [2]. The method has shown to yield good numerical solutions and in many respects it can be considered as a good representative of a large class of upwind discretization methods. (See [1] for a comparison between the present basic discretization method and various other discretization methods.) Following Lax [4], the basic method discretizes the Euler equations in the integral form

$$
\oint_{\partial \Omega^{*}}\left(f(q) n_{x}+g(q) n_{y}\right) d s=0,
$$

where $\partial \Omega^{*}$ is the boundary of $\Omega^{*}, \Omega^{*}$ being an arbitrary subdomain of the computational domain $\Omega$, and where $n_{x}$ and $n_{y}$ are the $x$ - and $y$-component of the outward unit normal at $\partial \Omega^{*}$. The Euler equations are discretized by requiring (1.5) to hold for each $\Omega_{i, j}$, i.e. each $i, j$-th quadrilateral finite volume in a disjunct division of $\Omega$, $\Omega=\cup \Omega_{i, j}$. Given a quadrilateral finite volume division, along $\partial \Omega_{i, j}$ the contour integrial in (1.5) consists of four cell face contributions, each with constant $n_{x}$ and $n_{y}$. Per cell face, the line integral is approximated by taking the flux functions $f(q)$ and $g(q)$ constant and by computing them with Osher's approximate Riemann solver [6]. Denoting the cell face between e.g. $\Omega_{i, j}$ and $\Omega_{i+i, j}$ by $\partial \Omega_{i+1 / 2, j}$ and the Riemann solver approximating $(f(q))_{i+1 / 2, j}$ and $(g(q))_{i+1 / 2, j}$ by $F\left(\left(q_{i+1 / 2, j}^{l}, q_{i+1 / 2, j}^{r}\right)\right.$ and $G\left(\left(q_{i+1 / 2, j}^{l}, q_{i+1 / 2, j}^{r}\right)\right.$, the integral along cell face $\partial \Omega_{i+1 / 2, j}$ can be written as

$$
\begin{gathered}
\left.\iint_{\partial+1 /, j}(q) n_{x}+g(q) n_{y}\right) d s= \\
{\left[F\left(q_{i+1 / 2, j}^{l}, q_{i+1 / 2, j}^{r}\right)\left(n_{x}\right)_{i+1 / 2, j}+G\left(q_{i+1 / 2, j}^{l}, q_{i+1 / 2, j}^{r}\right)\left(n_{y}\right)_{i+1 / 2, j}+O\left(h^{\mu}\right)\right] l_{i+1 / 2, j},}
\end{gathered}
$$

where $l_{i+1 / 2, j}$ denotes the length of $\partial \Omega_{i+1 / 2, j}$ and $\mu$ the order of accuracy of the approximation. On smooth grids, first-order accuracy $(\mu=1)$ is guaranteed by taking $q_{i+1 / 2, j}^{i}$ and $q_{i+1 / 2, j}^{r}$ equal to the states in the corresponding adjacent volumes. 
Boundary conditions are incorporated in a way which is consistent with the discretization in the interior of the domain. At subsonic inflow this requires the specification of three boundary conditions. At subsonic outflow, one boundary condition is required. (Just obeying these numbers still does not guarantee mathematical wellposedness. For a study of the mathematical well-posedness of e.g. some typical subsonic outlet boundary conditions, we refer to [3].) For supersonic flow the number of boundary conditions to be imposed at in- and outflow is four and zero, respectively.

In this paper we focus on fully subsonic Euler flows with constant entropy imposed at inflow, i.e. subsonic Euler flow problems of which the exact solutions are known to be homentropic. We will make proper use of this knowledge to monitor the quality of numerical solutions obtained for these flows.

\subsection{The problem}

First we study the error behaviour in the approximation of a homentropic, subsonic Euler flow along a smooth wall (Fig.1.1). The angle $\delta$ over which the wall has been bended (Fig.1.1) is $\delta=10^{\circ}$. For further details about this test case we refer to [5]. The type of grid used is given in Fig.1.2. It consists of $32 \times 32$ finite volumes. Coarser grids $(16 \times 16$ and $8 \times 8)$ are obtained from the finer by leaving out each second mesh line. In Fig.1.3 we show the entropy error along the continuously curved wall for the family of three grids mentioned. The behaviour of the entropy error is clearly first-order accurate; it vanishes at an $O(h)$-rate.

Next, we study the error behaviour in the approximation of the similar subsonic Euler flow along a kinked wall (Fig.1.4). (Except for the kink, all boundary conditions are identical to those in the smooth test case.) For the type of grid shown in Fig.1.5 we obtain the entropy error behaviour given in Fig.1.6. The entropy error is zeroth-order accurate; it does not vanish for $h \rightarrow 0$ ! Causes of the zeroth-order behaviour may be:

(i) non-smoothness of the grid (The grid in Fig.1.4 is non-smooth along the entire grid line emerging from the kink in the wall.), or

(ii) non-smoothness of the exact solution. (The incompressible potential flow along a kinked wall is known to have a singularity in the velocity field of the form $r^{\alpha}, 0<\alpha<1$, where $r$ denotes the radial distance from the kink and where $\alpha$ is a constant depending on the kink angle. The subsonic, compressible Euler flow may have a similar singularity.)

In the following we will investigate both possible causes. 


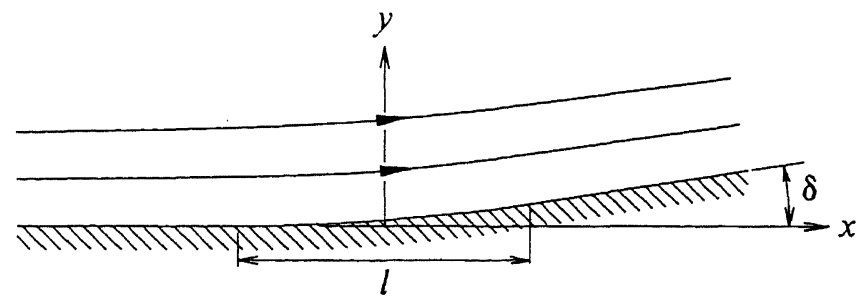

Fig.1.1. Smooth wall.

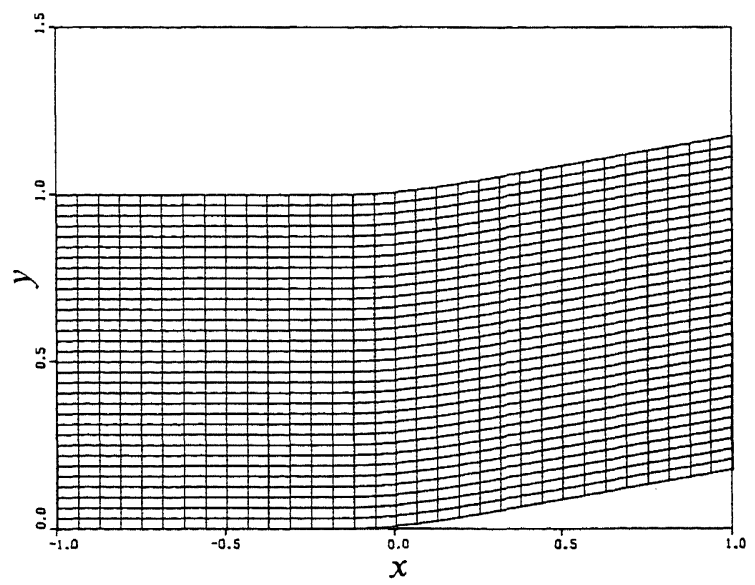

Fig.1.2. Smooth grid, $32 \times 32$.

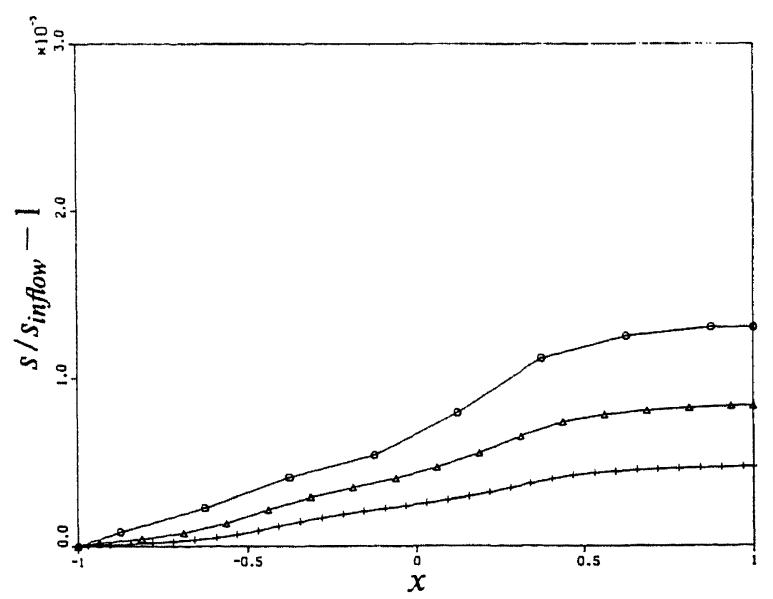

Fig.1.3. Entropy error along smooth wall, $\delta=10^{\circ}$, $0: 8 \times 8$-grid, $\Delta: 16 \times 16$-grid, $+: 32 \times 32$-grid. 


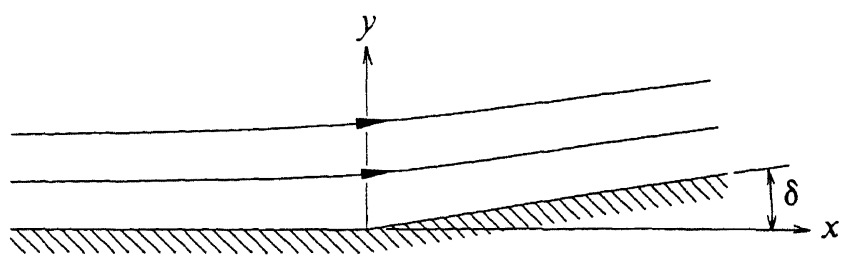

Fig.1.4. Kinked wall.

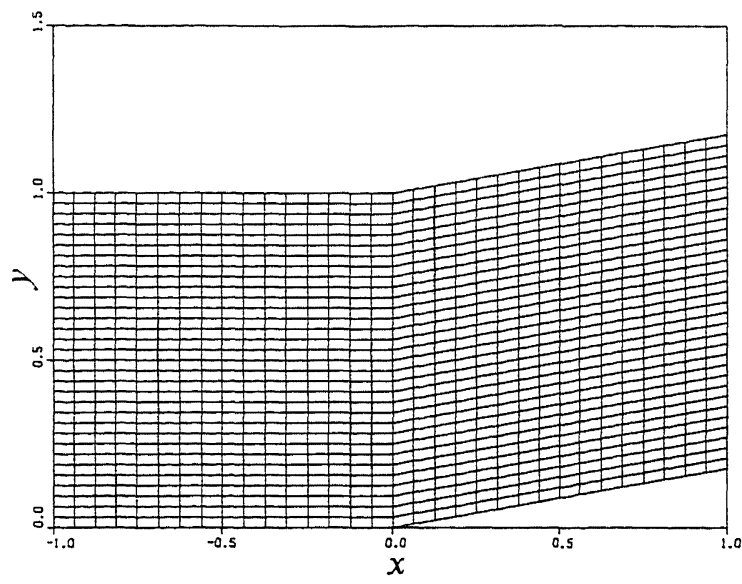

Fig.1.5. Kinked grid, $32 \times 32$.

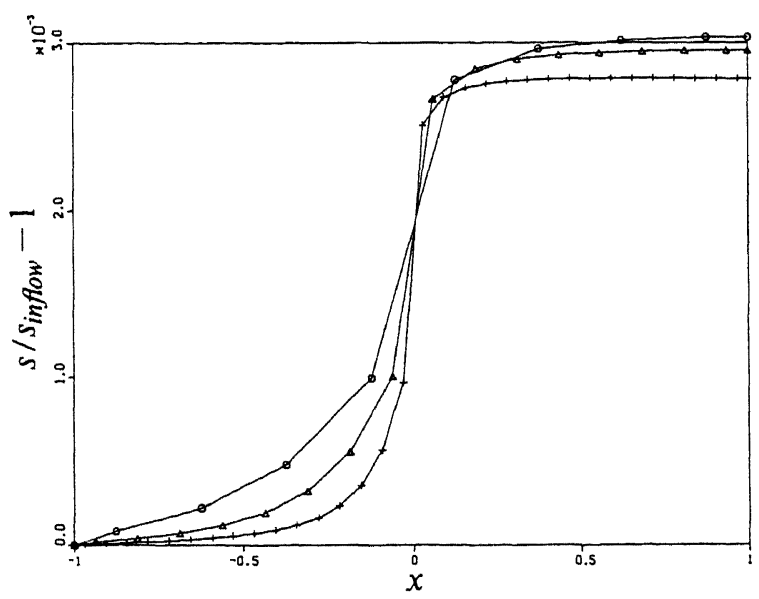

Fig.1.6. Entropy error along kinked wall, $\delta=10^{\circ}$, $0: 8 \times 8$-grid, $\Delta: 16 \times 16$-grid, $+: 32 \times 32$-grid. 


\section{NON-SMOOTHNESS OF THE GRID}

\subsection{Local discretization error}

Given the zeroth-order order error behaviour, it is natural first to investigate the discretization error in the upwind finite volume discretization on a kinked grid. For $\Omega_{i \star, j}$, a cell just behind the kink in the grid (Fig.2.1), the discretized equation reads

$$
\begin{gathered}
{\left[F\left(q_{i \star, j}, q_{i \star}+1, j\right)-F\left(q_{i \star-1, j}, q_{i \star, j}\right)\right]} \\
+2 \tan \delta\left[F\left(q_{i \star, j-1}, q_{i \star, j}\right)-F\left(q_{i \star, j}, q_{i \star, j}+1\right)\right] \\
+2\left[G\left(q_{i \star, j}, q_{i \star, j+1}\right)-G\left(q_{i \star, j-1}, q_{i \star, j}\right)\right]=0 .
\end{gathered}
$$

By performing Taylor series expansions around the centre of $\Omega_{i \star, j},(2.1)$ can be rewritten as

$$
\left.\frac{\partial F}{\partial x}\right|_{i \star, j}+\left.\frac{\partial G}{\partial y}\right|_{i \star, j}-\left.\frac{1}{2} \tan \delta\left[\frac{\partial F\left(q, q_{i \star, j}\right)}{\partial q} \frac{\partial q}{\partial y}\right]\right|_{i \star, j}=O(h) .
$$

We see that the discretization has a zeroth-order error. For $\delta=0$ or for fully onesided upwind discretization from the right, the zeroth-order error vanishes. (In the latter case $F\left(q, q_{i \star, j}\right)=f\left(q_{i \star, j}\right)$ and hence $\partial F\left(q, q_{i \star, j}\right) / \partial q=0$.) For a cell $\Omega_{i \star-1, j}$ just in front of the kink (Fig.2.1), we obtain the comparable result

$$
\left.\frac{\partial F}{\partial x}\right|_{i \star-1, j}+\left.\frac{\partial G}{\partial y}\right|_{i \star-1, j}+\left.\frac{1}{2} \tan \delta\left(\frac{\partial F\left(q_{i \star}-1, j, q\right)}{\partial q} \frac{\partial q}{\partial y}\right)\right|_{i \star-1, j}=O(h) .
$$

For $\Omega_{i \star-1, j}$, the zeroth-order error vanishes for a fully left-sided upwind discretization, or - again - for vanishing $\delta$. For further details and also a numerical verification of the analysis we refer to [5], where we compute the residual of an exact solution of the continuous Euler equations, by substituting this exact solution into the discrete Euler equations on a kinked grid.

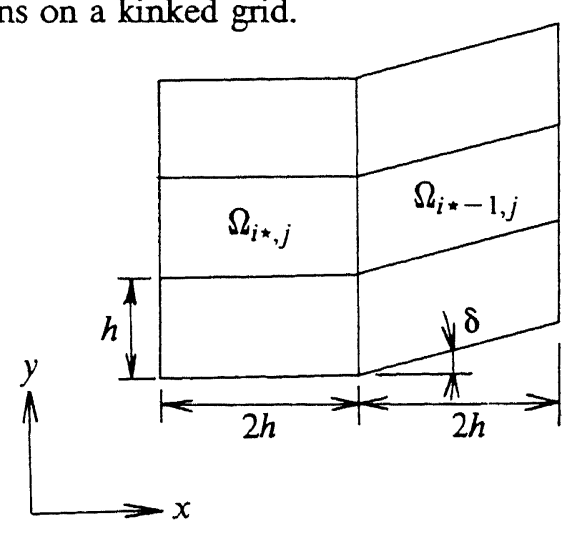

Fig.2.1 Geometry at kink. 


\subsection{New discretization}

The zeroth-order discretization errors found in (2.2) and (2.3), only involve a derivative in $y$-direction, i.e. the direction in which the cells behind the kink have been shifted. We will now try to remove these errors by enlarging the discretization stencil. Because we only need to introduce extra derivatives in $y$-direction, we expand the stencil by using additional information from $y$-direction. We introduce a new flux across $\partial \Omega_{i \star+1 / 2, j}$, by taking a weighted mean of the basic numerical fluxes across $\partial \Omega_{i \star+1 / 2, j}$ and $\partial \Omega_{i \star+1 / 2, j+1}$, i.e. we approximate $f_{i \star+1 / 2, j}$ by

$$
f_{i \star+1 / 2, j}=a_{i \star+1 / 2, j} F\left(q_{i \star, j}, q_{i \star+1, j}\right)+\left(1-a_{i \star+1 / 2, j}\right) F\left(q_{i \star, j+1}, q_{i \star+1, j+1}\right) .
$$

The same is done for the approximation of the flux across $\partial \Omega_{i \star-1 /, j}$ :

$$
f_{i \star-1 / 2, j}=a_{i \star-1 / 2, j} F\left(q_{i \star-1, j}, q_{i \star, j}\right)+\left(1-a_{i \star-1 / 2, j}\right) F\left(q_{i \star-1, j+1}, q_{i \star, j+1}\right) \text {. }
$$

Substitution of (2.4) and (2.5) into the discrete version of (1.5), then yields after Taylor series expansion around the centre of $\Omega_{i \star, j}$ :

$$
\begin{gathered}
\left.\frac{\partial F}{\partial x}\right|_{i \star, j}+\left.\frac{\partial G}{\partial y}\right|_{i \star, j} \\
+\left.\frac{1}{2}\left(a_{i \star-1 / 2, j}-a_{i \star+1 / 2, j}-\tan \delta\right) \frac{\partial F}{\partial y}\right|_{i \star, j}+\left.\frac{1}{2} \tan \delta\left(\frac{\partial F\left(q_{i \star, j}, q\right)}{\partial q} \frac{\partial q}{\partial y}\right)\right|_{i \star, j}=O(h) .(2.6)
\end{gathered}
$$

The third term in (2.6) equals zero by choosing the weights such that they satisfy $a_{i \star-1 / 2, j}-a_{i \star+1 / 2, j}-\tan \delta=0$. Given our upwind discretization, the fourth term automatically drops for a supersonic flow from the left.

As a numerical experiment we consider now, on a kinked grid without wall: a supersonic parallel flow with: $u=u_{0}+u_{1} y, u_{0}$ and $u_{1}$ being positive constants, and with: $v=0, \rho$ is constant and $p$ is constant. Using the old discretization, for this exact solution of the continuous Euler equations there is no zeroth-order error in $\Omega_{i \star-1, j}$ [5]. This already fixes $a_{i \star-1 /, j}$ at $a_{i \star-1 / 2, j}=1$. Next substituting the exact solution into the new discrete equations, we can make the zeroth-order error in $\Omega_{i \star, j}$ equal to zero by taking $a_{i \star+1 / 2, j}=1-\tan \delta$. (Notice that application of the new discretization in $\Omega_{i \star, j}$ introduces zeroth-order errors in the cells downstream of $\Omega_{i \star, j}$. In order to remove these errors, we apply the new discretization in all cells downstream of the kink.) In Fig.2.2 local discretization errors are given, as found with the new discretization and this exact solution of the continuous Euler equations. We observe that the error behaviour is first-order indeed.

In subsonic flow the Riemann solver is not fully one-sided and as a consequence, in subsonic flow the zeroth-order discretization error in (2.6) can be removed only by making the sum of the third and fourth term equal to zero. In that case the weights $a_{i \star-1 / 2, j}$ and $a_{i \star+1 / 2, j}$ become dependent of the solution. So, in subsonic flow the zeroth-order local discretization error cannot be removed without introducing a significantly more complicated discretization. Before possibly making any attempt to construct a discretization of this kind, in the next section we will first investigate, to what extent the zeroth-order discretization error actually causes the zeroth-order entropy error. 
a. On $8 \times 8$-grid.

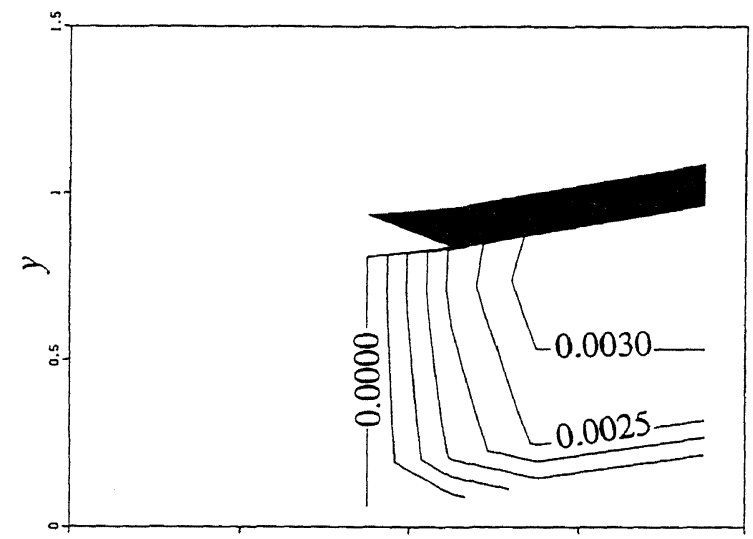

b. On $16 \times 16$-grid.

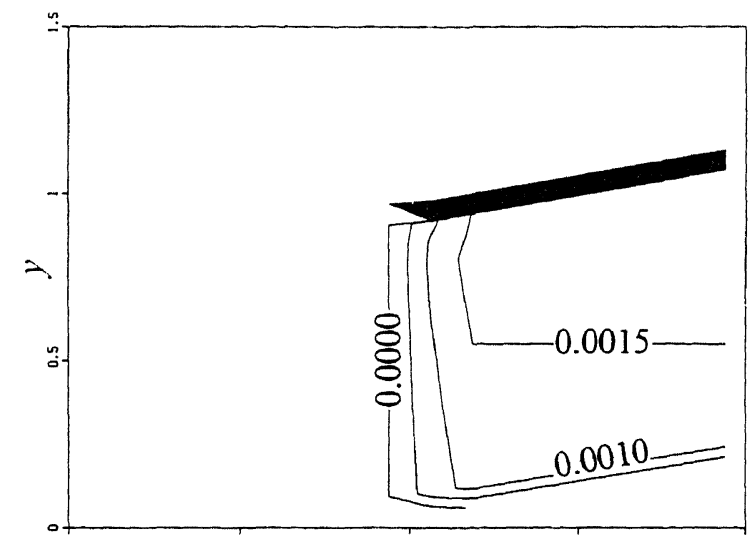

c. On $32 \times 32$-grid.

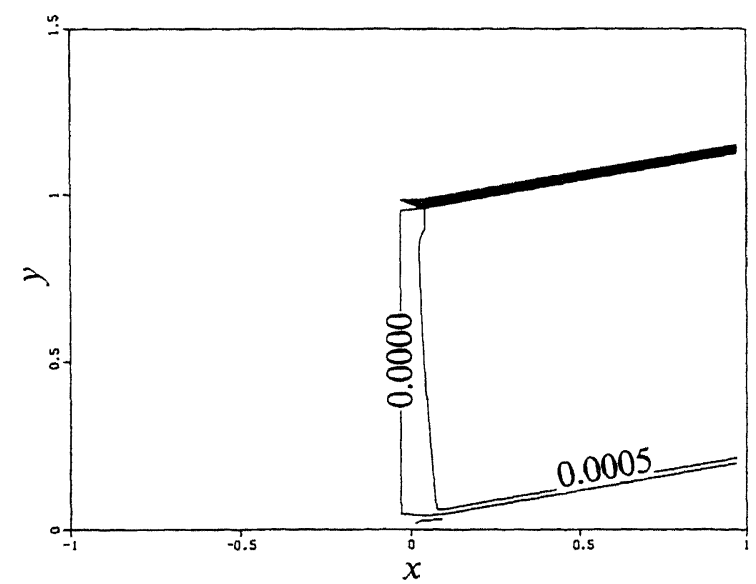

Fig.2.2. Discretization errors in energy equation, $\delta=10^{\circ}$, supersonic, non-uniform parallel flow from the left, new discretization. 


\subsection{Entropy error on a kinked grid not involving a wall}

In this section we study the entropy error on a kinked grid without wall, using again the old discretization and imposing via the boundary conditions: a subsonic version of the exact parallel flow considered in the previous section. If for the flow without wall, on the kinked grid shown in Fig.1.5, zeroth-order errors in the solution field are found, then they may be caused by the zeroth-order local discretization error. If we do not find zeroth-order errors in the solution, assuming that the parallel flow imposed (via the boundary conditions) is not too trivial, the kinked grid and the resulting zeroth-order discretization errors do not cause zeroth-order errors in the solution field.

In Fig.2.3 we present numerical results which show the entropy error in the bottom row of cells, for a family of three grids. The results clearly show that the entropy error becomes approximately twice as small when the mesh width is made twice as small. Therefore, we postulate that the zeroth-order entropy error found in the numerical approximation of subsonic Euler flow along a kinked wall is not caused by the zeroth-order discretization error in the neighbourhood of the kink in the grid.

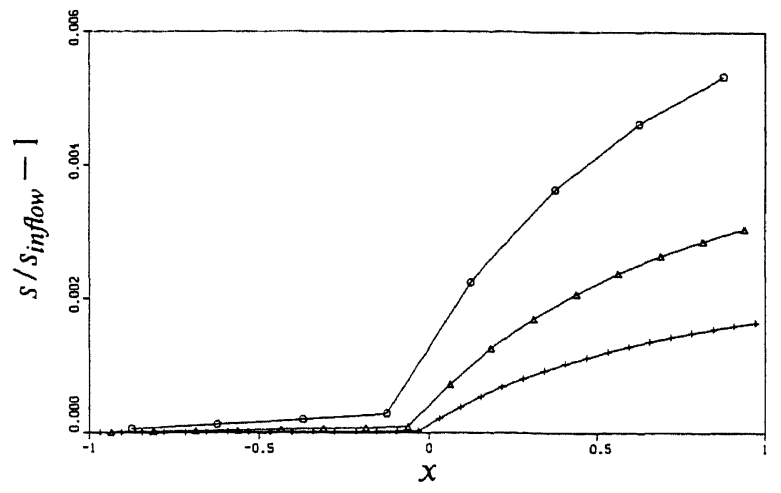

Fig.2.3. Entropy error in bottom row of cells, $\delta=10^{\circ}$, subsonic, non-uniform parallel flow from the left, old discretization, $0: 8 \times 8$-grid, $\Delta: 16 \times 16$-grid, $+: 32 \times 32$-grid. 


\section{SINGULARITY IN THE SOLUTION}

In this section we will consider another possible (and now most likely) cause of the error: a singularity in the solution at the kink.

\subsection{Irrotational, incompressible flow}

For the incompressible potential flow along a kinked wall the exact solution of the continuous potential flow equation is known. In the polar $r, \theta$-coordinates (Fig.3.1), for $\delta \leqslant \theta \leqslant \pi$, the velocity field is described by

$$
\begin{aligned}
& u=U r^{\frac{\delta}{\pi-\delta}} \cos \left(\theta-\frac{\theta-\delta}{\pi-\delta} \pi\right), \\
& v=U r^{\frac{\delta}{\pi-\delta}} \sin \left(\theta-\frac{\theta-\delta}{\pi-\delta} \pi\right),
\end{aligned}
$$

where $U$ is a constant. For $0<\delta<\pi / 2$, the velocity field has a singularity at $r=0$. (There, it is not differentiable.) In [5] we also compute this incompressible potential flow, using the compressible Euler equations extended with proper source term. The numerical solution obtained clearly shows a zeroth-order accuracy at the kink in the wall, a zeroth-order accuracy just as that found for the compressible Euler flow along a kinked wall. Since the incompressible potential flow is singular at the kink, from this we conclude that the subsonic, compressible Euler flow may be singular at the kink as well.

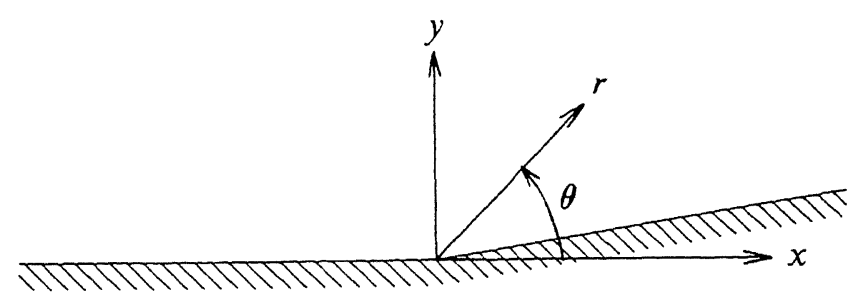

Fig.3.1. Polar coordinates at kink. 


\subsection{Transformation of variables and equations}

In this section we investigate whether we can transform the dependent variables in such a way that only smooth (i.e. non-singular) functions remain to be approximated. Herewith, we strive for a minor modification of the existing numerical method. We assume that the velocity field of the subsonic compressible Euler flow along a kinked wall has a singularity similar to that of the incompressible potential flow. In the previous section it was suggested that if this is the proper form of the singularity, the approximate solution, obtained by solving the discretized Euler equations, should have a zeroth-order error behaviour. By extracting now such a singularity, we hope to remove this behaviour. Assume that $u$ and $v$ can be written as

$$
\begin{aligned}
& u=U r^{\alpha}, \\
& v=V r^{\alpha},
\end{aligned}
$$

where $U$ and $V$ are smooth functions of $x$ and $y$ and where $\alpha=\delta /(\pi-\delta), 0<\delta<\pi / 2$. Further, assume that the exact Euler flow is homentropic and isenthalpic. Then, along the wall we have for the speed of sound

$$
c^{2}=c_{0}^{2}-\frac{\gamma-1}{2}\left(U^{2}+V^{2}\right) r^{2 \alpha}=C_{0}^{2}-C_{1}^{2} r^{2 \alpha}
$$

with $C_{0}$ a constant and $C_{1}$ a smooth function. If we assume that the enthalpy changes smoothly from one streamline to another, then (3.3) is valid everywhere in the flow field, with $C_{0}$ and $C_{1}$ smooth functions of $x$ and $y$. Furthermore, $C_{1}$ is a known function of $U$ and $V$. With $s=p / \rho^{\gamma}$ constant, we find for the density

$$
\rho=(\gamma s)^{\frac{-1}{\gamma-1}}\left(C_{0}^{2}-C_{1}^{2} r^{2 \alpha}\right)^{\frac{1}{\gamma-1}}=\left(R_{0}-R_{1} r^{2 \alpha}\right)^{\frac{1}{\gamma-1}},
$$

and for the pressure

$$
p=\left(\gamma^{\gamma} s\right)^{\frac{-1}{\gamma-1}}\left(C_{0}^{2}-C_{1}^{2} r^{2 \alpha}\right)^{\frac{\gamma}{\gamma-1}}=\left(P_{0}-P_{1} r^{2 \alpha}\right)^{\frac{\gamma}{\gamma-1}} .
$$

In this, $R_{0}, R_{1}, P_{0}$ and $P_{1}$ are smooth functions of $x$ and $y$, and $R_{1}$ and $P_{1}$ are known functions of $U$ and $V$. Next we can transform the Euler equations (1.1)-(1.2) into equations to be satisfied by our new, smooth variables $U, V, R_{0}, P_{0}$. The transformed fluxes are complicated, nonlinear functions [5]. The corresponding system of discretized equations cannot be solved by the existing method nor by a slightly modified version of it. Apart from the fact that the transformed equations require an ambitious modification of the existing numerical method, it is not yet certain whether or not the assumptions made on the singularity are correct. Therefore, we refrain from a further investigation of these equations. 


\section{GETTING ROUND THE ENTROPY ERROR BY PROPERLY ROUNDING THE KINK}

In section 1.3 we computed the Euler flow along a continuously curved wall. There, the length $l$ of the curved wall segment (Fig.1.1) is the same for all three grids considered. In this section we compute the flow along a continuously curved wall for which $l=O\left(h^{\lambda}\right), \lambda>0$. For finite $\lambda$, it is clear that for $h \rightarrow 0$ the wall becomes kinked. The results presented in Fig.1.3 and Fig.1.6 can be considered as those for the limit cases $\lambda=0$ and $\lambda=\infty$, respectively. (For $\lambda=\infty$, for all $h$, the continuously curved part of the wall is reduced to a single point and the entropy error is zeroth-order.) The number of cells $N$ lying along the curved part of the wall is $N=l / h$. Hence, with $l=O\left(h^{\lambda}\right)$ we have $N=O\left(h^{\lambda-1}\right)$. So, for $\lambda<1, N$ increases with decreasing $h$ and for $\lambda>1, N$ decreases. In the latter case, for $h \rightarrow 0$ we find that at the kink (which is in fact the curved part of the wall), there is only one mesh line. In that case we would have the same situation as for $\lambda=\infty$, the situation with zeroth-order entropy error. So, in order to have decreasing entropy errors for decreasing mesh size, we must take $\lambda<1$.

In Fig.4.1 now, we show the behaviour of the entropy error, when $l$ is decreased as a function of $h$. Herewith, for $l$ we take $l=c_{1} h^{\lambda}$ with $c_{1}$ constant, and for the maximum norm of the entropy error we assume the form $\left\|s / s_{\text {inflow }}-1\right\|_{\infty}=c_{2} h^{\mu}, c_{2}$ constant. Based on numerical experiments with $c_{1}=1, \mu$ has been determined for different values of $\lambda$, using a $16 \times 16$-, a $32 \times 32$ - and a $64 \times 64$-grid. As expected, for $\lambda=0$ we find that $\mu \rightarrow 1$ for $h \rightarrow 0$. The main message of Fig.4.1 is that the flow along a continuously curved wall can be used indeed, to approximate the flow along a kinked wall. For any $\lambda \in(0,1)$, in the limit $h \rightarrow 0$, the curved wall becomes kinked and the error vanishes, because for any $\lambda$ in that range it appears that $\left\|s / s_{\text {inflow }}-1\right\|_{\infty}=O\left(h^{\mu}\right), \mu>0$. (If we want to let the entropy error disappear at the same rate as $l$, then from Fig.4.1 we find that we should take $\lambda \approx 0.4$.)

Fig.4.1. Order behaviour maximal entropy error, $\delta=10^{\circ}$, properly rounded kink, $0: 16 \times 16$-grid $/ 32 \times 32$-grid, $\Delta: 32 \times 32$-grid $/ 64 \times 64$-grid.

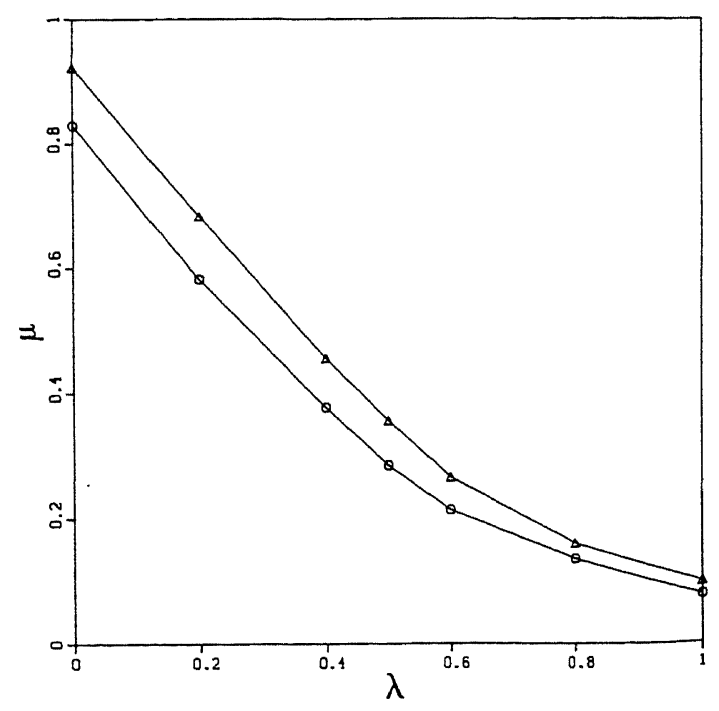




\section{Conclusions}

In this paper we studied two possible causes of zeroth-order entropy errors, as encountered in the numerical approximation of the subsonic Euler flow along a kinked wall: (i) non-smoothness of the grid and (ii) non-smoothness of the exact solution of the continuous Euler equations. (The computation of the flow along a smooth wall shows, as expected, that the entropy error is not caused by the discretization of the solid wall boundary condition.)

Concerning the non-smoothness of the grid, it appears that the upwind finite volume discretization of the equations has a zeroth-order local discretization error at the kink in the grid. For supersonic flow this discretization error can be removed by simply extending the stencil of the discretization in the direction in which the grid has been shifted. For subsonic flow a zeroth-order discretization error remains. However, the numerical approximation of a subsonic, non-uniform, parallel flow on a kinked grid without wall, suggests that the zeroth-order behaviour of the local discretization error does not cause the zeroth-order entropy error.

Concerning the non-smoothness (i.e. the being singular) of the exact solution, it is well-known that the incompressible potential flow along a kinked wall has a singularity at the kink. It is most likely that a similar singularity is adopted by the Euler flow. The numerical approximation of the incompressible potential flow, by means of the Euler equations with proper source term, shows that zeroth-order errors in the solution arise at the same places as in the approximation of the compressible Euler flow. Extraction of a singularity from the dependent variables of the Euler equations, a singularity which is similar to that of the incompressible potential flow solution, does not lead to a set of equations which can be solved with the existing method.

Removing the zeroth-order error behaviour is not impossible. We find the paradoxical result that by making a less accurate (but specific) discretization of the geometry, a numerical solution with better error behaviour can be obtained. The less accurate discretization of the geometry employs smooth discrete versions of the exact kinked geometry. The flow along a smooth wall is used to approximate the flow along a kinked wall. By making the length of the curved part of the smooth wall dependent of the mesh size $h$, an $O\left(h^{\mu}\right)$-entropy error, $\mu>0$, still can be obtained.

\section{ACKNOWLEDGEMENT}

The authors wish to thank Professor Hemker and Professor Wesseling for their constructive comments.

\section{REFERENCES}

1. A. Dervieux, B. van Leer, J. Périaux and A. Rizzi (eds.), Proc. GAMMWorkshop on the Numerical Simulation of Compressible Euler Flows, Rocquencourt, 1986; Notes on Numerical Fluid Dynamics, Vol. 26, Vieweg, Braunschweig, 1989.

2. P.W. Hemker and S.P. Spekreijse, 'Multiple grid and Osher's scheme for the efficient solution of the steady Euler equations', Appl. Numer. Math., 2, 475-493 (1986). 
3. B. Koren, 'Euler flow solutions for transonic shock wave - boundary layer interaction', Int. J. Numer. Methods in Fluids, 9, 59-73 (1989).

4. P.D. Lax, 'Hyperbolic systems of conservation laws II', Comm. Pure Appl. Math., 10, 537-566 (1957).

5. H.T.M. van der Maarel and B. Koren, 'Spurious entropy generation in a nonsmooth geometry', (submitted to Int. J. Numer. in Fluids).

6. S. Osher and F. Solomon, 'Upwind difference schemes for hyperbolic systems of conservation laws', Math. Comput., 38, 339-374 (1982). 